\title{
Capillary forces in tapping mode atomic force microscopy
}

\author{
L. Zitzler, S. Herminghaus, and F. Mugele* \\ Abteilung Angewandte Physik, Universität Ulm, D-89069 Ulm, Germany \\ (Received 3 May 2002; revised manuscript received 21 August 2002; published 31 October 2002)
}

\begin{abstract}
We investigated the influence of the relative humidity on amplitude and phase of the cantilever oscillation while operating an atomic force microscope (AFM) in the tapping mode. If the free oscillation amplitude $A_{0}$ exceeds a certain critical amplitude $A_{c}$, the amplitude- and phase-distance curves show a transition from a regime with a net attractive force between tip and sample to a net repulsive regime. For hydrophilic tip and sample this critical amplitude $A_{c}$ is found to increase with increasing relative humidity. In contrast, no such dependence was found for hydrophobic samples. Numerical simulations show that this behavior can be explained by assuming the intermittent formation and rupture of a capillary neck in each oscillation cycle of the AFM cantilever.
\end{abstract}

DOI: 10.1103/PhysRevB.66.155436

\section{INTRODUCTION}

More and more, atomic force microscopy is used to study not only the surface topography, but also the local mechanical properties and the chemical composition of samples. ${ }^{1-3}$ The so-called tapping mode has become the most widely used operation mode, in particular when dealing with soft samples such as polymers, ${ }^{4,5}$ biological samples, ${ }^{6}$ or liquids. ${ }^{7}$ In order to extract the mechanical properties of the surface, it has become popular to record amplitude-phase-distance (APD) curves, where the amplitude and phase of the oscillating cantilever are measured while the average distance $d_{0}$ between tip and sample is continuously reduced (or increased). ${ }^{1,8-10}$ At large separation, the cantilever is well represented by a simple harmonic oscillator, whereas the interaction with the substrate adds a nonlinear contribution to the potential of the cantilever as the (average) tip-sample distance $d_{0}$ is reduced. ${ }^{11}$ Through this nonlinearity, material properties such as adhesion, elasticity, or viscoelasticity influence the cantilever dynamics. In parallel to the experiments, a significant amount of theoretical studies and numerical simulations were performed to elucidate how these properties can be extracted from APD curves. ${ }^{12-17}$ The characteristic features of the cantilever dynamics, such as shifts of the resonance frequency, the existence of oscillation regimes with predominantly attractive or predominantly repulsive tip-sample interaction were properly explained and their relation to sample properties could be established in many cases. Experiments related to soft matter, such as polymers or biological samples, are typically carried out in ambient atmosphere. Under these conditions, the ambient humidity usually leads to a thin film of water covering both tip and sample. ${ }^{18}$ If the AFM cantilever comes close to the sample surface a capillary neck may form between tip and sample. ${ }^{19}$ While it was shown in the past that capillary forces have a strong impact on force-distance curves in conventional (contact-mode) $\mathrm{AFM},{ }^{20-22}$ little attention was paid to their influence on the dynamics of an AFM cantilever in the tapping mode. Considering the strength of the capillary forces, which is typically in the $\mathrm{nN}$ range, one would expect to find their footprint in tapping mode APD curves. In this paper we present a combined experimental and numerical simulation study of the AFM cantilever dynamics at variable relative humidity (RH). First (Sec. II) we present experimental APD
PACS number(s): 68.37.Ps, 68.08.Bc

curves at variable humidity on both hydrophilic and hydrophobic samples. In Sec. III, we describe our model for the dynamics of the tip/sample ensemble in the presence of a hysteretic capillary force. Section IV is devoted to the results of the numerical simulations. In Sec. V, we discuss the results and their relation to typical experimental situations when imaging soft samples in the presence of adsorbed water layers.

\section{EXPERIMENTAL RESULTS}

\section{A. Experimental details}

Our experiments were performed at room temperature with a "MultiMode" AFM from Digital Instruments equipped with a NanoScope III controller. We used conventional tapping mode cantilevers made of $\mathrm{Si}$ with a resonance frequency of $f_{0} \approx 280 \mathrm{kHz}$. We prepared hydrophilic and hydrophobic substances of both Si and mica. Hydrophilic Si samples were cleaned in ultrasonic baths of acetone, toluene and ethanol and stored overnight in an oxidizing solution of NOCHROMIX (Ref. 23) in concentrated sulfuric acid. The tips were rinsed with acetone, toluene, and ethanol. Hydrophilic mica samples were cleaved immediately prior to the experiment. Hydrophobic surfaces of tips and samples (both mica and $\mathrm{Si}$ ) were obtained by exposing them to a saturated vapor atmosphere of heptadecafluoro-1,1,2,2-tetrahydrodecyldimethylchlorosilane (PFS) for 1 to $2 \mathrm{~h}$. Excess PFS was washed away with ethanol. The advancing water contact angle on the hydrophobic samples was $\approx 115^{\circ}$. On the hydrophilic samples it was less than $5^{\circ}$. Both cantilevers and samples were mounted to the AFM and allowed to equilibrate for a few hours. The experiments were carried out at an operating frequency $f$ equal to the resonance frequency $f_{0}$ of the free cantilever. To control the environmental conditions, we closed the front side of the sample and cantilever compartment of the instrument with a piece of plexiglass. A gentle and continuous stream of $\mathrm{N}_{2}$ was blown into this chamber. The relative humidity of the $\mathrm{N}_{2}$ stream could be adjusted between 3 and $96 \%$ by controlling the mixing ratio between a dry and a wet $\left(\mathrm{H}_{2} \mathrm{O}\right.$-saturated) stream of $\mathrm{N}_{2}$ gas.

\section{B. Amplitude- and phase-distance curves}

Figure 1 shows two typical experimental APD curves for a hydrophilic tip on a hydrophilic Si sample. Each APD 

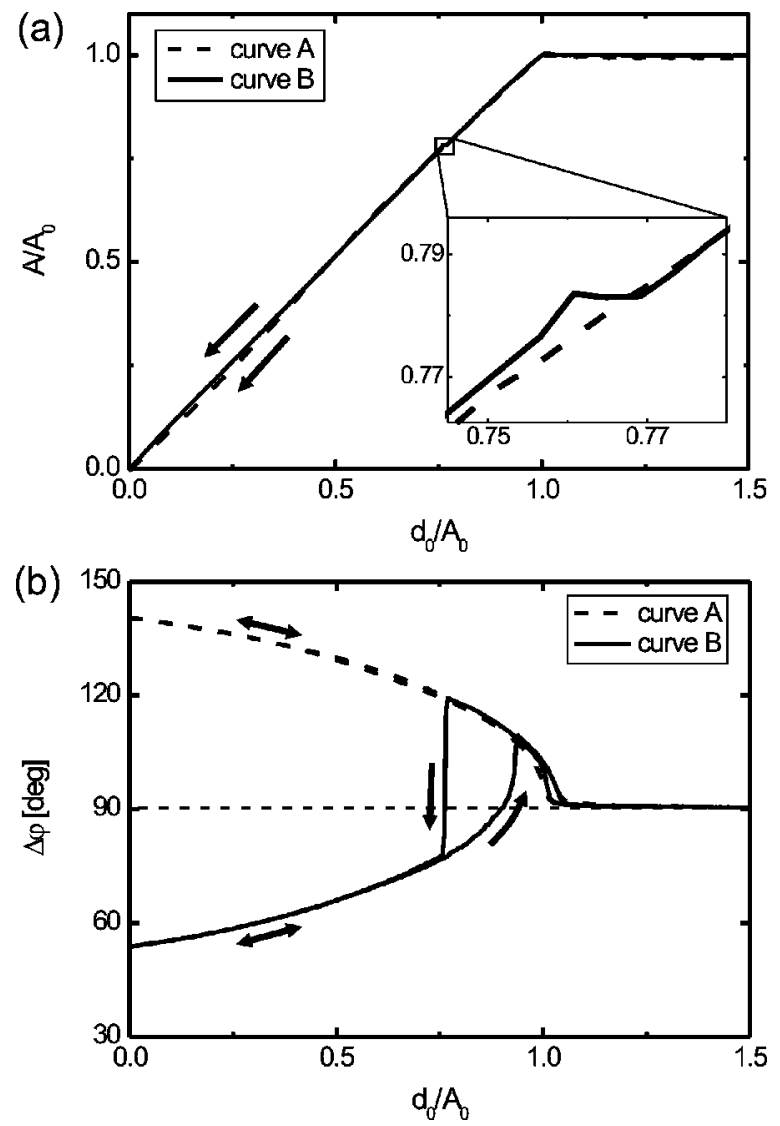

FIG. 1. (a) Amplitude- and (b) phase-distance curves. The free oscillation amplitudes were $A_{0}=37 \mathrm{~nm}$ (curve A) and $A_{0}=46 \mathrm{~nm}$ (B). $\mathrm{RH} \approx 50 \%$ for both curves. For clarity the retracting parts of the APD curves are omitted in (a) (they lie within the error bars of the approaching curves).

curve was recorded by automatically reducing the average distance $d_{0}$ between tip and sample from some initial value $d_{0}$ with $d_{0}>A_{0}=A\left(d_{0}=\infty\right)$ down to a minimum value of $d_{0, \text { min }}$ such that the measured oscillation amplitude $A\left(d_{0, \min }\right)$ was reduced to $20 \%$ of the free amplitude $A_{0}$. Initially, we had allowed for a hard mechanical contact between tip and sample $\left(d_{0, \min } \approx 0\right)$. However, it turned out that the reproducibility of the APD curves was poor in this case, in particular for the hydrophobic surfaces. We attributed these effects to irreversible modifications of either the tip or the sample surface or both. Therefore, all the data presented here refer to the range $d_{0} \geqslant d_{0, \text { min }}$ such that $A\left(d_{0, \text { min }}\right)=0.2 A_{0}$.

The scaling of the ordinate of the amplitude-distance curve [Fig. 1(a)] was done by locating the intersection between the horizontal line (for $d_{0} / A_{0}>1$ ) and the linearly descending line (for $d_{0} / A_{0}<1$ ) with slope 1 . This point corresponds to the absolute value of $A_{0}$ on the $d_{0}$-axis (and of course on the $A$-axis, too). The scaling of the $\varphi$-axis in Fig. 1(b) was done by converting the output of the microscope software to real values of the phase shift. ${ }^{24,25}$

Curve $\mathrm{A}$ in Fig. 1 was recorded with a free oscillation amplitude $A_{0}=37 \mathrm{~nm}$, whereas for curve B $A_{0}$ was set to 46 $\mathrm{nm}$, with otherwise identical parameters. For curve A the amplitude of the cantilever oscillation decreases continuously as $d_{0}$ is reduced (slope $\approx 1$ ). The relative phase shift
$\Delta \varphi$ (with respect to large separations) is positive throughout the whole range of average tip-sample separation. It was shown previously that a positive phase shift corresponds to an overall attractive tip-sample interaction (averaged over an entire oscillation cycle). Under these conditions, the APD curves for decreasing and for increasing average tip-sample separation are identical within the experimental error. For curve $\mathrm{B}$, however, $\Delta \varphi$ is positive for large $d_{0}$, but it becomes negative below a certain critical value $d_{0, c}$. At the same separation, there is a small discontinuity in the cantilever amplitude. For the retracting branch of the APD curve $d_{0, c}$ is larger than for the approaching branch. In the experimental literature, it is common practice to denote deviations between the two branches as "hysteresis." However, one should be aware that this kind of deviation is not necessarily related to dissipation. It can also be obtained in completely conservative systems. ${ }^{8,12}$ The deviations reflect the existence of two oscillation modes of the anharmonic oscillator, which is bistable in certain regions of the phase space. ${ }^{16,26}$ The appearance of the repulsive regime depends on a variety of AFM operating parameters. It is well known ${ }^{15}$ that one of the most important ones is the free amplitude $A_{0}$ at large separations, which measures the total energy stored in the cantilever oscillation. For sufficiently low $A_{0}$, all the APD curves look similar to curve $A$. The transition to the repulsive regime is suppressed essentially all the way down to $d_{0} \approx 0$. When $A_{0}$ exceeds a critical (threshold) value $A_{c}$, however, there is a transition to the repulsive regime at some critical average separation $d_{0, c}\left(A_{0}\right)$. Experimentally, we measured $A_{c}$ by increasing $A_{0}$ in equidistant steps $\Delta A_{0}$ (beginning at small $A_{0}$ ) and recorded APD curves such that we had at least five curves of either type, $\mathrm{A}$ or $\mathrm{B}$. Thus $A_{c}$ is located between the maximum value of $A_{0}$, for which an APD curve of type A was recorded and the minimum value of $A_{0}$, for which an APD curve of type B was recorded.

\section{Humidity dependence of $\boldsymbol{A}_{\boldsymbol{c}}$}

At first glance, the data presented in Fig. 1 do not show any signature that can be attributed to the presence of an adsorbed water film. To study the latter in more detail, we recorded APD curves and extracted the value of $A_{c}$ for various values of RH. Experimentally, we adjusted the humidity of the $\mathrm{N}_{2}$ stream and allowed the system to equilibrate for 5 min. After this waiting time the APD curves did not change any more within the experimental error. For each value of RH we chose a new position on the sample to avoid any possible continuous degradation over the time of the measurement. Furthermore we readjusted the drive frequency to the resonance frequency $f_{0}$ for each RH. $f_{0}$ decreased by approximately $15 \mathrm{~Hz}$ from the lowest to the highest humidity. This is in agreement with the expected mass uptake of the cantilever upon water adsorption. Then, we recorded APD curves as described above, beginning with small amplitudes. Qualitatively, all the APD curves look strikingly similar, independent of the humidity and both for hydrophilic and hydrophobic samples. In contrast to what one might have anticipated, we cannot make out any indication of cap- 

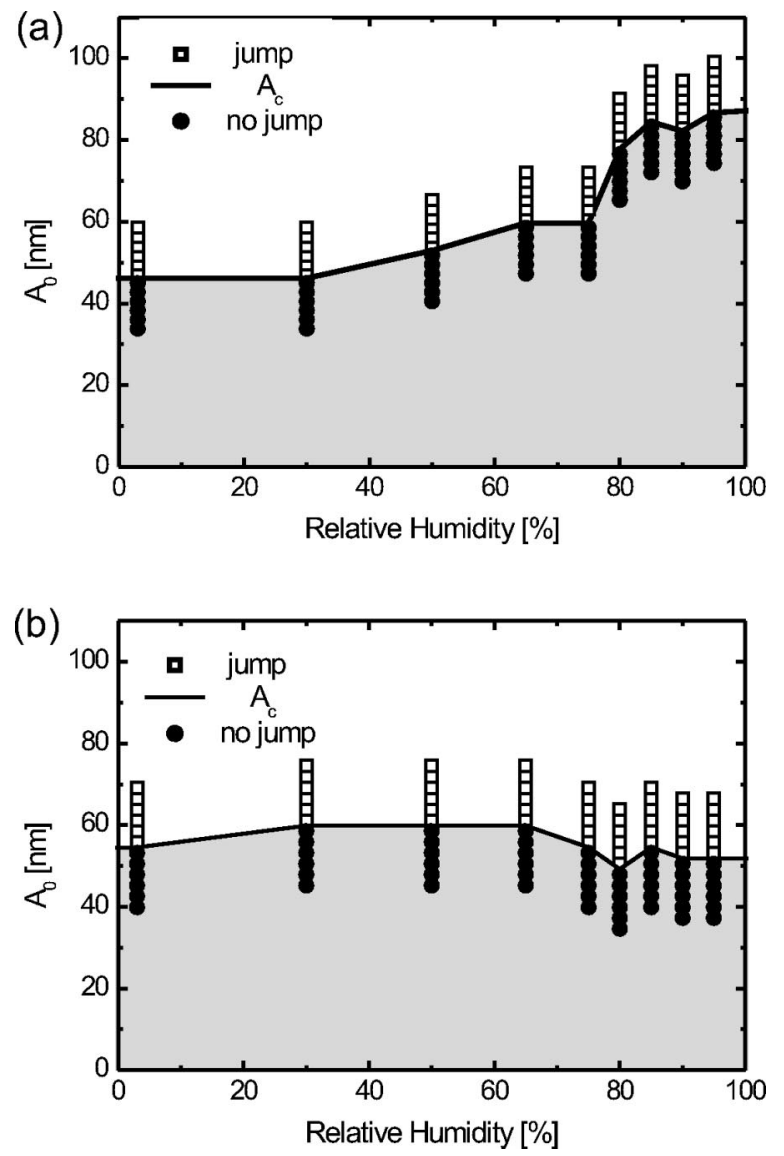

FIG. 2. Influence of the relative humidity on the oscillation amplitude $A$. The circles (open squares) indicate experimental APD curves without (with) a jump to the repulsive regime, i.e., APD curves of type A (type B) in Fig. 1. While for hydrophilic tip and sample $A_{c}$ increases with RH (a), no such dependence could be observed for a hydrophobic sample (b).

illary neck formation directly in the individual APD curves. The situation is different, however, if we look at the onset of the repulsive regime.

Figure 2(a) shows a strong increase of $A_{c}$ with increasing humidity for a combination of a hydrophilic tip and a hydrophilic sample. Compared to its value under dry conditions, $A_{c}$ almost doubled upon approaching 100\% RH. Apparently, the main impact of the adsorbed water layer is to increase $A_{c}$. In order to prove that this effect is really due to the tip/sample interaction, we repeated the same experiment on a hydrophobic sample. In this case, there is no significant dependence on the relative humidity [Fig. 2(b)]. Thus the increase shown in Fig. 2(a) must be due to the adsorbed water layer. This is the main experimental finding of the present work. Qualitatively, we reproduced the same behavior for hydrophilic and hydrophobic mica samples.

\section{MODEL}

\section{A. Equation of motion}

To understand this behavior in detail, we simulated the cantilever oscillation numerically. Figure 3 shows a sketch of a vibrating cantilever/tip ensemble above a substrate.

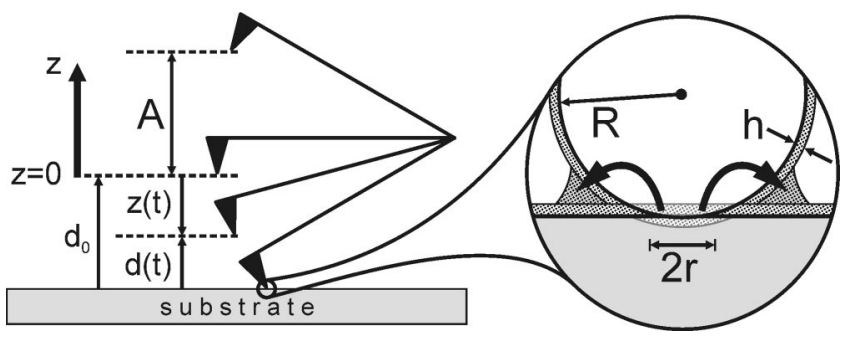

FIG. 3. Model of a vibrating cantilever/tip ensemble near a substrate. The two coordinates $z$ and $d$ are shown as well as the thin layer of adsorbed water at the sample and the tip. When tip and sample are in contact the water in the overlapping region is displaced and accumulated at the edge of the contact area. This amount of water (with volume $V$ ) is assumed to form a meniscus when the tip is retracted.

Several other authors ${ }^{13,14}$ have shown that the dynamical behavior of the tip can be described as a driven anharmonic oscillator:

$$
m \ddot{z}+\frac{m \omega_{0}}{Q} \dot{z}+k_{c} z-F_{\mathrm{ts}}(d)=F_{0} \cos (\omega t) .
$$

$m, \omega_{0}, Q, k_{c}$ are the effective (point) mass, the angular resonance frequency, the (constant) quality factor, and the spring constant of the cantilever/tip ensemble, respectively. $F_{\text {ts }}=F_{\text {ts }}(d)$ denotes the distance dependent tip-sample interaction, which consists of the van der Waals force $F_{\mathrm{vdW}}$, the elastic repulsive force $F_{\text {rep }}$, and the capillary forces $F_{\text {cap }} . F_{0}$ and $\omega(=2 \pi f)$ are the amplitude and angular frequency of the external sinusoidal driving force, respectively.

\section{B. Van der Waals and repulsive forces}

The tip is modeled by a sphere of radius $R$ whereas the sample is assumed to be a flat (infinite radius of curvature). The first contribution to the tip-sample interaction $F_{\text {ts }}$ is the long-range attractive van der Waals force. For the sphere-flat geometry $F_{\mathrm{vdW}}$ is

$$
F_{\mathrm{vdW}}=\left\{\begin{aligned}
-\frac{H R}{6 d^{2}}, & d>a_{0}, \\
-\frac{H R}{6 a_{0}^{2}}, & d \leqslant a_{0},
\end{aligned}\right.
$$

where $H$ is the Hamaker constant. $a_{0}$ is an intermolecular distance which is introduced to avoid the divergence of $F_{\mathrm{vdW}}$ for $d \rightarrow 0 . a_{0}$ can be obtained by assuming that for separations $d \leqslant a_{0}$ the van der Waals force $F_{\mathrm{vdW}}$ can be replaced by the adhesion force $F_{\text {adh }}$ given by the DMT (DerjaguinMuller-Toporov) model of contact mechanics: ${ }^{27,28}$

$$
F_{\mathrm{adh}}=-4 \pi R \gamma_{\mathrm{SV}}=-\frac{H R}{6 a_{0}^{2}} \Leftrightarrow a_{0}=\sqrt{\frac{H}{24 \pi \gamma_{\mathrm{SV}}}} .
$$

Here, $\gamma_{\mathrm{SV}}$ is the solid-vapor interfacial energy for both tip and sample. 
The second contribution to $F_{\text {ts }}$ is the elastic repulsive force $F_{\text {rep }}$ which acts only during contact if $d \leqslant a_{0}$. For relatively stiff samples (such as $\mathrm{Si}$ ) this force is described best by the DMT theory: ${ }^{28}$

$$
F_{\text {rep }}=\frac{4}{3} E^{*} \sqrt{R}\left(a_{0}-d\right)^{3 / 2}, d \leqslant a_{0}
$$

with the effective Young's molulus

$$
\frac{1}{E^{*}}=\frac{1-\nu_{\mathrm{tip}}^{2}}{E_{\mathrm{tip}}}+\frac{1-\nu_{\text {sample }}^{2}}{E_{\text {sample }}}
$$

where $E_{\text {tip }}\left(E_{\text {sample }}\right)$ and $\nu_{\text {tip }}\left(\nu_{\text {sample }}\right)$ are the Young's modulus and the Poisson coefficient of the tip and the sample, respectively.

Numerical models that include only these two (conservative) contributions to $F_{\text {ts }}$ are well established. ${ }^{13,14}$ However, simple functions such as (2) or (4) are not suitable to describe the capillary interaction because they do not allow for hysteresis.

\section{Capillary force}

As a first approach to the problem, we tried to keep the capillary force model as simple as possible. The "zoomed view" in Fig. 3 shows the geometry of the tip/sample ensemble. Both tip and sample are covered by a thin water film of thickness $h$. When tip and sample are in contact the water in the overlapping region is displaced and accumulated at the edge of the contact area. This amount of water (with volume $V$ ) is assumed to form a meniscus when the tip is retracted. A simple geometric model leads to a meniscus volume of

$$
V_{\mathrm{men}}=4 \pi R h^{2}+\frac{4}{3} \pi h^{3}+2 \pi r^{2} h
$$

with a circular contact area of radius $r$. The first two terms on the right hand side of Eq. (6) describe the overlapping volume of the water layers for an undeformed sphere in contact with a plane. The last term accounts for the displaced volume within the contact area when the sphere is elastically deformed (see Fig. 3). Using the DMT theory and negligible load the radius $r$ of the contact area is ${ }^{28}$

$$
r=\sqrt[3]{\frac{3 \pi \gamma_{\mathrm{SV}} R^{2}}{E^{*}}} .
$$

The capillary force shows a strong hysteresis. Consider an oscillating AFM cantilever. Initially, at large separation, there is no capillary neck. Upon approaching the surface, a meniscus forms at a certain separation $d_{\text {on }}$. If we neglect instabilities due to van der Waals attraction or capillary condensation, the meniscus forms upon geometrical contact of the adsorbed water layers, i.e., when $d_{\text {on }}=2 h$. Upon retracting the tip the meniscus breaks at a distance $d_{\text {off }}>d_{\text {on }}$. Willet et al. ${ }^{29}$ give a formula for this rupture distance as a function of the radius of curvature of the sphere and of the contact angle. The formula is a best-fit approximation to numerical

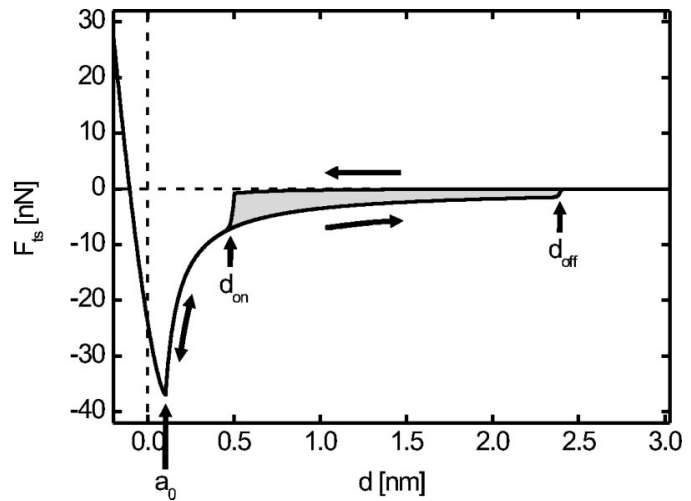

FIG. 4. Distance dependence of the force $F_{\mathrm{ts}}(d)$ between tip and sample, which occurs in every single oscillation. One clearly sees the hysteretic character of $F_{\text {ts }}$ which is due to the capillary force $F_{\text {cap }}(R=20 \mathrm{~nm}, h=0.2 \mathrm{~nm})$. The gray area in between the approaching and retracting curve represents the energy which is dissipated in each oscillation cycle due to the nonconservative character of $F_{\text {cap }}$.

solutions of the Laplace-Young equation. In our case, the contact angle $\theta$ is zero both on the tip and on the sample. We obtain

$$
d_{\mathrm{off}}=V^{1 / 3}-\frac{1}{5 R} V^{2 / 3}
$$
force $^{30}$

When the meniscus is present, we add the capillary

$$
F_{\text {cap }}=-\frac{4 \pi \gamma_{\mathrm{H}_{2} \mathrm{O}} R}{1+d / h}
$$

to the total tip-sample force. $\gamma_{\mathrm{H}_{2} \mathrm{O}}$ is the liquid-vapor interfacial energy of water. For simplicity, we keep $F_{\text {cap }}$ constant for $d \leqslant a_{0}$. Summing up all the contributions, the total tip-

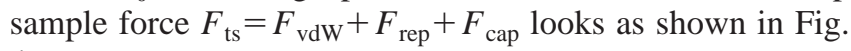
4.

Note two different aspects of this plot: First, there is the hysteresis between the approaching and the retracting branch of the force curve. Whenever the cantilever amplitude is large enough, a capillary neck forms at $d=d_{\text {on }}$ and breaks again at $d=d_{\text {off }}$ within each cantilever oscillation cycle. This gives rise to an energy dissipation per cycle corresponding to the area shaded in light gray. Second, in the range between $d_{\text {on }}$ and $d_{\text {off }}$, the capillary force is significantly larger than the van der Waals force. The area under the hysteresis loop represents a substantial fraction of the total integral over the force curve over a full oscillation cycle.

\section{NUMERICAL SIMULATIONS}

\section{A. Parameters and details of the numerical calculation}

Our simulations were performed for a $\mathrm{Si}$ tip and a $\mathrm{Si}$ sample. We used a Hamaker constant of $H=6.0 \times 10^{-20} \mathrm{~J}$, an effective Young's modulus of $E^{*}=65 \mathrm{GPa}$ ( $E_{\text {tip }}$ $\left.=E_{\text {sample }} \approx 120 \mathrm{GPa}, \nu_{\text {tip }}=\nu_{\text {sample }}=0.3\right)$ and a surface energy of $\gamma_{\mathrm{SV}}=75 \mathrm{~mJ} / \mathrm{m}^{2}$. The surface energy of water is $\gamma_{\mathrm{H}_{2} \mathrm{O}}$ 
$=72 \mathrm{~mJ} / \mathrm{m}^{2}$. For the cantilever-tip ensemble we chose tip radii of $R=20 \mathrm{~nm}$ and $R=50 \mathrm{~nm}$, a quality factor of $Q$ $=400$, a spring constant of $k_{c}=27.5 \mathrm{~N} / \mathrm{m}$, and a resonance frequency of $f_{0}=280 \mathrm{kHz}$. This corresponds to an effective mass of $m=k_{c} / \omega_{0}^{2}=8.9 \times 10^{-12} \mathrm{~kg}$. All simulations were performed for $f=f_{0}$. The last parameter which is necessary to compare the simulations to experiments is the amplitude $F_{0}$ of the driving force. It was adjusted by matching the cantilever amplitude at large separation, i.e., outside the range of $F_{\text {ts }}$, where the tip/cantilever ensemble is a simple harmonic oscillator. To calculate the full APD curves, we solved Eq. (1) using a standard fourth-order Runge-Kutta algorithm. ${ }^{31}$ For each value of the average separation $d_{0}$, the solution showed a transient oscillation which decayed into the steady state within a few $\tau=2 Q / \omega_{0}$. The amplitude $A$ and the phase $\varphi$ were extracted from the steady oscillation by the fast Fourier transformation. Each value of $A$ and $\varphi$ served as initial condition for amplitude and phase of the next point in the APD curve. In agreement with earlier findings, both experimental and theoretical, ${ }^{32}$ a plot of the amplitude vs frequency shows that the amplitude of the second harmonic frequency is at most $5 \times 10^{-3}$ of the amplitude at the fundamental frequency. The motion of the cantilever is thus reasonably well described by a simple harmonic oscillation. During the simulations it turned out that the numerical algorithm was not able to deal with abrupt steps in the force curve, as they occur upon neck formation or rupture. Therefore, we introduced a continuous function to interpolated between the pure van der Waals force and the van der Waals force plus capillary force (see Fig. 4). For the simulation results presented in this work, the transition region had a width of $0.05 \mathrm{~nm}$. We checked that a reduction of this width to $0.01 \mathrm{~nm}$ does not influence our results.

\section{B. Numerically simulated APD curves}

The above model was used to calculate amplitude- and phase-distance curves for various values of water film thickness $h$. Figure 5 shows two phase-distance curves $\left(A_{0}\right.$ $=20 \mathrm{~nm}$ for curve A; $A_{0}=21 \mathrm{~nm}$ for curve B) for a film thickness of $h=0.2 \mathrm{~nm}$.

For curve A in Fig. 5(b), $\Delta \varphi>0$ for all values of the normalized average separation $d_{0} / A_{0}$. Within the numerical resolution the approaching and retracting branches are identical. For a free amplitude of only $1 \mathrm{~nm}$ more (curve B), there was a transition from positive to negative values of $\Delta \varphi$ at some $d_{0, c}$. As in the experimental curves, the simulated cantilever dynamics switched from the branch of predominantly attractive tip-sample interaction to the one of overall repulsive interaction. Upon increasing $d_{0}$, the switch back from the repulsive to the attractive branch occurred at a somewhat larger separation. This hysteresis confirms the experimental results (see Fig. 1) and demonstrates the bistability of the nonlinear oscillator for this specific range of parameters. The amplitude-distance curve is shown in Fig. 5(a): As in the experimental curves there is an almost linear reduction of the amplitude with decreasing tip-sample separation $d_{0} / A_{0}$. If the free oscillation amplitude $A_{0}$ exceeds $A_{c}$ one can observe a minor jump to a higher amplitude.
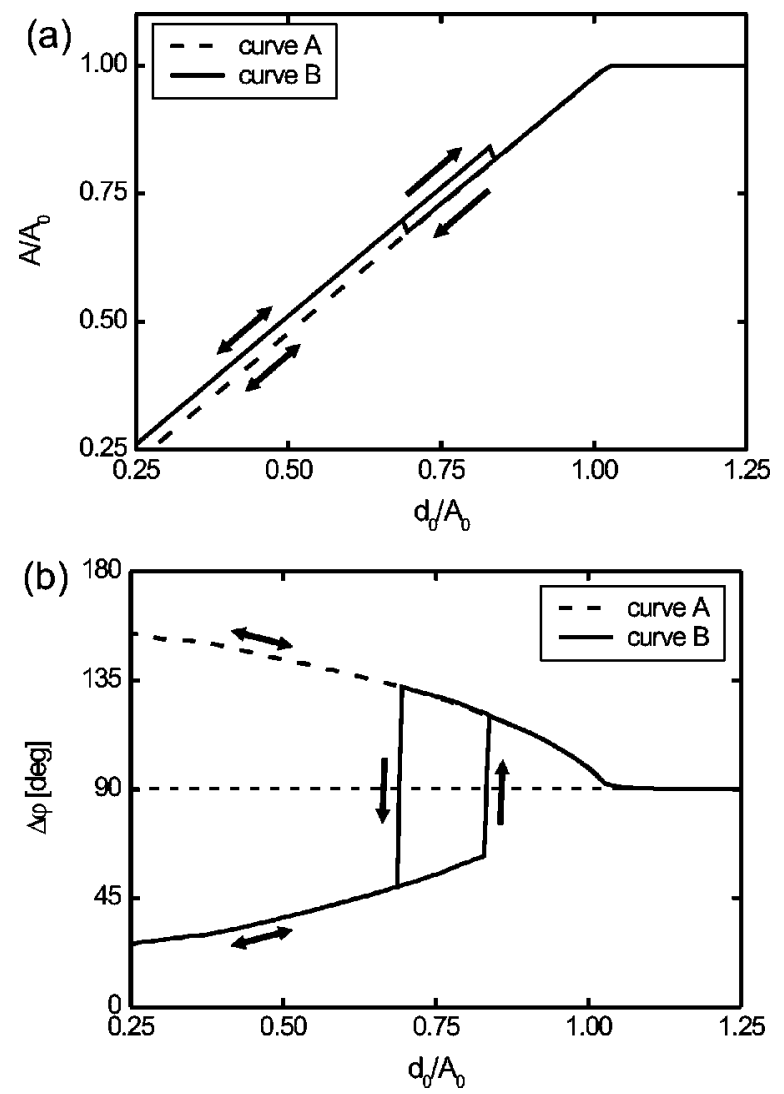

FIG. 5. Simulated APD curve for two different free oscillation amplitudes $A_{0}=20 \mathrm{~nm}$ (curve A) and $A_{0}=21 \mathrm{~nm}$ (curve B). While curve $\mathrm{A}\left(A_{0}<A_{c}\right)$ shows no jump to the repulsive regime and therefore no hysteresis, the retracting part of curve $\mathrm{B}\left(A_{0}>A_{c}\right)$ remains significantly longer in the repulsive regime than the approaching part. For even larger $A_{0}$ the jumps to and from the repulsive regime occur at larger values of $d_{0} / A_{0}$ and the width of the hysteresis is smaller. Here the critical amplitude would be $A_{c}=(20.5$ $\pm 0.5) \mathrm{nm}$.

The numerical data thus reproduce all the experimental results, including the fact that there is no obvious feature in the APD curves that can be related directly to the capillary force. However, there is one direct consequence that can be extracted: The formation and rupture of the capillary neck leads to additional energy dissipation. If we neglect the contribution of higher harmonics, we can calculate the average power dissipation due to the tip-sample interaction: ${ }^{33}$

$$
\bar{P}_{\mathrm{diss}}=\frac{k_{c} A^{2} \omega_{0}}{2 Q}\left(\frac{A_{0}}{A} \sin \varphi-1\right) .
$$

$A$ and $\varphi$ are the amplitude and phase of the oscillation at a certain distance $d_{0} . A_{0}$ is the free oscillation amplitude as defined previously.

The upper solid curve in Fig. 6 shows the result for a typical simulation run (see Fig. 5) in the presence of a water film with thickness $h=0.2 \mathrm{~nm}$.

At large separation, the dissipation is zero as expected. At small separation, there is a plateau with an average power dissipation of $\bar{P}_{\text {diss }} \approx 1.25 \mathrm{pW}$. The width of the smooth tran- 


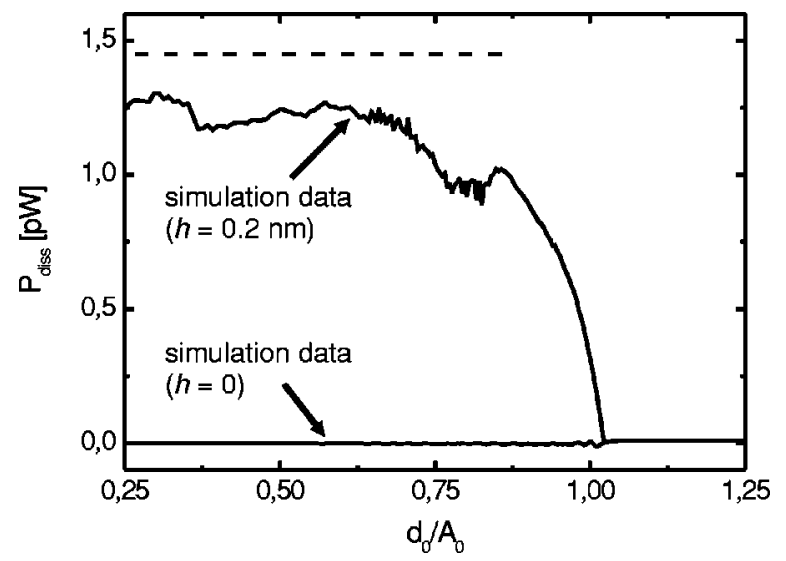

FIG. 6. Average dissipated power due to the tip-sample interaction. The upper solid curve shows $\bar{P}_{\text {diss }}$ for the simulated APD curves shown in Fig. 5 which uses $F_{\text {ts }}$ from Fig. 4 . If no capillary force is included no dissipation occurs (lower solid curve). The dashed line gives the expected value based on the area of the hysteresis loop in Fig. 4.

sition region $\left(0.85<d_{0} / A_{0}<1.02\right)$ is related to the smooth onset of the capillary force in our simulations. From the product of the area of the hysteresis loop (Fig. 4) and the resonance frequency, we obtain a slightly larger value for the expected dissipation of $\bar{P}_{\text {diss }} \approx 1.4 \mathrm{pW}$. We attribute the minor deviation to higher harmonics, which begin to be excited as soon as the meniscus formation sets in. For comparison, we also plot the dissipation for a simulation without any water (lower solid curve). As expected, the dissipation scatters only weakly around zero in this case.

To quantify the impact of the water film thickness $h$ on the critical amplitude $A_{c}$, we increased $h$ systematically from 0 to $2.25 \mathrm{~nm}$. For each value of $h$, we performed simulations for various values of $A_{0}$ separated by steps of $1 \mathrm{~nm}$ and monitored the first occurrence of a transition to the repulsive regime. The inset of Fig. 7 shows $A_{c}(h)$ for two different values of the tip radius, namely, $R=20 \mathrm{~nm}$ and $R=50 \mathrm{~nm}$.

The simulation data in Fig. 7 reproduce the experimental observation: An increase of the film thickness $h$ results in a

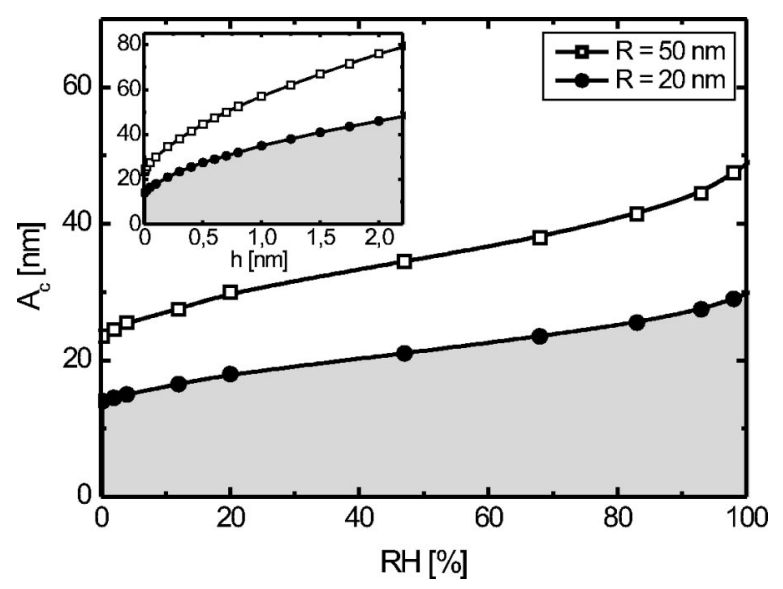

FIG. 7. Dependence of the critical oscillation amplitude $A_{c}$ on the thickness of the adsorbed water layer (inset) and on the relative humidity (large figure). See text for details. larger critical oscillation amplitude $A_{c}$ (inset). To compare the numerical data with the experiments one has to convert the film thickness $h$ to relative humidity. In order to do so, we used adsorption isotherms measured earlier by Beaglehole and Christenson ${ }^{18}$ using ellipsometry. They found a maximum thickness of $0.7 \mathrm{~nm}$ of water adsorbed on $\mathrm{Si}$ at $100 \%$ RH. The main part of Fig. 7, where $A_{c}$ is plotted versus RH, thus covers only the data points from the inset within $0 \leqslant h \leqslant 0.7 \mathrm{~nm}$. Note that $h$ depends on $\mathrm{RH}$ in a strongly nonlinear fashion. The agreement between the simulation results and the experimental data is satisfactory. The simulations reproduce the overall increase in $A_{c}$ by approximately a factor of 2 from the completely dry to the very humid limit. Figure 7 also shows that the absolute value of $A_{c}$ increases with increasing tip radius. Qualitatively, this is what we expect, because the capillary force is proportional to the tip radius. We simulated APD curves as a function of the tip radius for three different values of humidity $(20,70$, $100 \%)$. We found that $A_{c}$ scales as with $R^{0.55 \pm 0.01}$ over three decades of tip radius. Within the error, the exponent does not depend on the humidity. Presently, we have no explanation for this remarkable behavior.

\section{DISCUSSION}

The qualitative agreement between simulations and experiments indicates that the basic assumption of our model, namely, the formation and rupture of a capillary neck in each cantilever oscillation cycle, is correct. Despite the simplicity of the capillary model, even the absolute value of $A_{c}$ in the simulations is reasonably close to the experimental one. In fact, the agreement could be improved by adjusting some poorly defined parameters. In particular, $A_{c}$ is very sensitive to the cutoff length $a_{0}$ of the continuum elasticity model. A quantitative comparison of experiments and simulations would require a more careful characterization of the cantilever spring constant, the tip radius, and the wettability of the tip. It would be interesting to explore if one can extract information about dynamics in the adsorbed water layer from the measurements. In reality, the volume of the capillary neck does not remain constant between its formation and its rupture. Within its lifetime of a few hundred nanoseconds, its shape is likely to evolve owing to capillary condensation from the gas phase $\mathrm{e}^{34,35}$ and lateral transport in the liquid layer. The contribution of the latter effect increases for thicker liquid layers. In particular, we believe that AFM imaging of bulk liquids, which is becoming more and more popular for fundamental studies of wetting, ${ }^{7,36-38}$ is also based on the repeated formation and rupture of a capillary neck. This idea was proposed earlier and backed up by energetic arguments. ${ }^{7,39}$ The dynamic model described here could eventually lead to a quantitative understanding of the imaging process. In this case, we expect the hydrodynamics of neck formation and rupture to play a crucial role.

The role of the capillary neck in our model is slightly different from the one suggested by Baró et al. in a series of papers. ${ }^{19,40,41}$ They also concluded from their experiments that a capillary neck forms between tip and sample at sufficiently small separation. However, these authors attributed 
dissipation to the viscosity of the adsorbed water film. We do not believe that this mechanism dominates in our experiments. Viscous dissipation inside the contact zone was studied in detail for viscoelastic polymer samples, where close agreement between experiments ${ }^{42-44}$ and simulations ${ }^{45}$ was obtained. The dissipated power is typically on the order of a few tens of $\mathrm{pW}$. The viscous dissipation inside an aqueous capillary neck would involve similar velocities, however the viscosity of water is at least three or four orders of magnitude smaller, depending on the glass temperature of the polymer. Therefore the viscous dissipation should also be three to four orders of magnitude smaller than in the polymer case, i.e., it should be on the order of tens of fW. This value is negligible compared to the $\mathrm{pW}$ dissipation due to the nonconservative character of the capillary force (see Fig. 6).

Our results are also interesting in the context of local mechanical spectroscopy of soft materials. The quantitative measurement of local elastic or viscoelastic properties, the detailed analysis of the local indentation depth, ${ }^{3,42}$ or of the local work of adhesion, is certainly affected by the capillary forces discussed in this work. In particular, neglecting capillary forces might lead to false interpretations if the thickness of the water layer varies between different locations on the sample due to gradients in wettability. Such situations arise, for instance, in the case of phase-separated polymer blends or bloc copolymers. On the other hand, lateral variations of the capillary force also offer an opportunity to image the distribution of hydrophilic and hydrophobic areas on a surface. The capillary force mechanism could be particularly interesting for biological applications, where the recognition of different species is frequently based on their hydrophilic or hydrophobic nature.

\section{CONCLUSION}

We showed experimentally that increasing the ambient humidity suppresses the jump from the attractive to the repulsive oscillation mode in the tapping mode APD curves on hydrophilic Si samples. The critical free oscillation amplitude $A_{c}$ of the cantilever, which is required to observe the transition, was found double between the lowest and the highest relative humidity investigated. This behavior was explained in a model based on the intermittent formation of a capillary neck between tip and sample close to the lower turning point of the cantilever oscillation. The difference between the tip-sample separation where the capillary neck forms and the one where it ruptures, gives rise to a hysteretic capillary force. The latter provides the mechanism of energy dissipation in our model. Numerical simulations reproduced the doubling of $A_{c}$ with increasing humidity and thus lend credibility to the capillary force model. We expect that this model will lead to a quantitative understanding of the tapping mode imaging of bulk liquids. Furthermore, it should provide a way to characterize the local wettability on samples with laterally varying hydrophobicity, as frequently encountered in biology.

\section{ACKNOWLEDGMENTS}

We gratefully acknowledge the Deutsche Forschungsgemeinschaft for funding this work as part of the SFB 569 ("Hierarchic Structure Formation and Function of OrganicInorganic Nano Systems").
*Electronic address: frieder.mugele@ physik.uni-ulm.de

${ }^{1}$ S. Kopp-Marsaudon, P. Leclère, F. Dubourg, R. Lazzaroni, and J. P. Aimé, Langmuir 16, 8432 (2000).

${ }^{2}$ G. Bar, Y. Thomann, R. Brandsch, H.-J. Cantow, and M.-H. Whangbo, Langmuir 13, 3807 (1997).

${ }^{3}$ A. Knoll, R. Magerle, and G. Krausch, Macromolecules 34, 4159 (2001).

${ }^{4}$ R. Seemann, S. Herminghaus, and K. Jacobs, J. Phys.: Condens. Matter 13, 4925 (2001).

${ }^{5}$ R. Magerle, Phys. Rev. Lett. 85, 2749 (2000).

${ }^{6}$ H. G. Hansma, Annu. Rev. Phys. Chem. 52, 71 (2001).

${ }^{7}$ T. Pompe, A. Fery, and S. Herminghaus, Langmuir 14, 2585 (1998).

${ }^{8}$ X. Chen, M. C. Davies, C. J. Roberts, S. J. B. Tendler, P. M. Williams, and N. A. Burnham, Surf. Sci. 460, 292 (2000).

${ }^{9}$ Q. Ouyang, K. Ishida, and K. Okada, Appl. Surf. Sci. 169-170, 644 (2001).

${ }^{10}$ R. G. Winkler, J. P. Spatz, S. Sheiko, M. Möller, P. Reinecker, and O. Marti, Phys. Rev. B 54, 8908 (1996).

${ }^{11}$ J. P. Spatz, S. Sheiko, M. Möller, R. G. Winkler, and O. Marti, Nanotechnology 6, 40 (1995).

${ }^{12}$ B. Anczykowski, D. Krüger, and H. Fuchs, Phys. Rev. B 53, 15 485 (1996).

${ }^{13}$ N. A. Burnham, O. P. Behrend, F. Oulevey, G. Gremaud, P.-J. Gallo, D. Gourdon, E. Dupas, H. M. P. A. J. Kulik, and G. A. D.
Briggs, Nanotechnology 8, 67 (1997).

${ }^{14}$ R. García and A. S. Paulo, Phys. Rev. B 60, 4961 (1999).

${ }^{15}$ R. García and A. S. Paulo, Ultramicroscopy 82, 79 (2000).

${ }^{16}$ R. García and A. S. Paulo, Phys. Rev. B 61, R13 381 (2000).

${ }^{17}$ F. Gissibl, Phys. Rev. B 56, 16010 (1997).

${ }^{18}$ D. Beaglehole and H. K. Christenson, J. Phys. Chem. 96, 3395 (1992).

${ }^{19}$ J. Colchero, A. Storch, M. Luna, J. G. Herrero, and A. M. Baró, Langmuir 14, 2230 (1998).

${ }^{20}$ M. He, A. S. Blum, D. E. Aston, C. Buenviaje, R. M. Overney, and R. Luginbühl, J. Chem. Phys. 114, 1355 (2001).

${ }^{21}$ T. Stifter, O. Marti, and B. Bhushan, Phys. Rev. B 62, 13667 (2000).

${ }^{22}$ X.-D. Xiao and L. Qian, Langmuir 16, 8153 (2000).

${ }^{23}$ NOCHROMIX is an inorganic oxidizing glass cleaning reagent from Godax Laboratories, Inc.

${ }^{24}$ D. Bocek (unpublished).

${ }^{25}$ The DI NanoScope III controller uses the so called Bocek degrees $\varphi_{\text {Bocek }}$. They are converted to real degrees $\varphi$ by $\varphi$ $=\arccos \left(\varphi_{\text {Bocek }} / 90^{\circ}\right)$ (Ref. 24).

${ }^{26}$ M. Marth, D. Maier, J. Honerkamp, R. Brandsch, and G. Bar, J. Appl. Phys. 85, 7030 (1999).

${ }^{27}$ B. V. Derjaguin, V. M. Muller, and Y. P. Toporov, J. Colloid Interface Sci. 53, 314 (1975).

${ }^{28}$ W. N. Unertl, J. Vac. Sci. Technol. A 17, 1779 (1999). 
${ }^{29}$ C. D. Willett, M. J. Adams, S. A. Johnson, and J. P. K. Seville, Langmuir 16, 9396 (2000).

${ }^{30}$ J. N. Israelachvili, Intermolecular and Surface Forces, 2nd ed. (Academic, London, 1991).

${ }^{31}$ The simulations were performed with MATLAB Version 6.0, Release 12 from The MathWorks, Inc.

${ }^{32}$ R. García and T. Rodríguez, Appl. Phys. Lett. 80, 1646 (2002).

${ }^{33}$ J. P. Cleveland, B. Anczykowski, A. E. Schmid, and V. B. Elings, Appl. Phys. Lett. 72, 2613 (1998).

${ }^{34}$ M. M. Kohonen, N. Maeda, and H. K. Christenson, Phys. Rev. Lett. 82, 4667 (1999).

${ }^{35}$ M. M. Kohonen and N. Maeda (unpublished).

${ }^{36}$ T. Pompe and S. Herminghaus, Phys. Rev. Lett. 85, 1930 (2000).

${ }^{37}$ S. D. A. Connell, S. Allen, C. J. Roberts, J. Davies, M. C. Davies, S. J. B. Tendler, and P. M. Williams, Langmuir 18, 1719 (2002).
${ }^{38}$ S. Haferl, D. Poulikakos, and Z. Zhao, Exp. Heat Transfer 14, 1 (2001).

${ }^{39}$ S. Herminghaus, T. Pompe, and A. Fery, J. Adhes. Sci. Technol. 14, 1767 (2000).

${ }^{40}$ M. Luna, J. Colchero, and A. M. Baró, Appl. Phys. Lett. 72, 3461 (1998).

${ }^{41}$ A. Gil, J. Colchero, M. Luna, J. G. Herrero, and A. M. Baró, Langmuir 16, 5086 (2000).

${ }^{42}$ G. Bar, L. Delineau, R. Brandsch, M. Bruch, and M.-H. Whangbo, Appl. Phys. Lett. 75, 4198 (1999).

${ }^{43}$ G. Bar, R. Brandsch, M. Bruch, L. Delineau, and M.-H. Whangbo, Surf. Sci. Lett. 444, L11 (2000).

${ }^{44}$ G. Bar, L. Delineau, R. Brandsch, M. Ganter, and M.-H. Whangbo, Surf. Sci. 457, L404 (2000).

${ }^{45}$ A. S. Paulo and R. García, Surf. Sci. 471, 71 (2001). 